\title{
Analysis of Dependence Tracking Algorithms for Task Dataflow Execution
}

\author{
HANS VANDIERENDONCK, GEORGE TZENAKIS, and \\ DIMITRIOS S. NIKOLOPOULOS, Queen's University Belfast
}

Processor architectures has taken a turn toward many-core processors, which integrate multiple processing cores on a single chip to increase overall performance, and there are no signs that this trend will stop in the near future. Many-core processors are harder to program than multicore and single-core processors due to the need for writing parallel or concurrent programs with high degrees of parallelism. Moreover, many-cores have to operate in a mode of strong scaling because of memory bandwidth constraints. In strong scaling, increasingly finer-grain parallelism must be extracted in order to keep all processing cores busy.

Task dataflow programming models have a high potential to simplify parallel programming because they alleviate the programmer from identifying precisely all intertask dependences when writing programs. Instead, the task dataflow runtime system detects and enforces intertask dependences during execution based on the description of memory accessed by each task. The runtime constructs a task dataflow graph that captures all tasks and their dependences. Tasks are scheduled to execute in parallel, taking into account dependences specified in the task graph.

Several papers report important overheads for task dataflow systems, which severely limits the scalability and usability of such systems. In this article, we study efficient schemes to manage task graphs and analyze their scalability. We assume a programming model that supports input, output, and in/out annotations on task arguments, as well as commutative in/out and reductions. We analyze the structure of task graphs and identify versions and generations as key concepts for efficient management of task graphs. Then, we present three schemes to manage task graphs building on graph representations, hypergraphs, and lists. We also consider a fourth edgeless scheme that synchronizes tasks using integers. Analysis using microbenchmarks shows that the graph representation is not always scalable and that the edgeless scheme introduces least overhead in nearly all situations.

Categories and Subject Descriptors: D.1.3 [Programming Techniques]: Parallel Programming; D.3.2 [Programming Languages]: Dataflow Languages; C.1.4 [Processor Architectures]: Parallel Architectures

General Terms: Design, Algorithms, Performance

Additional Key Words and Phrases: Task dataflow, scheduling

New Article, Not an Extension of a Conference Paper.

The authors wish to express their gratitude to Giorgis Giorgakoudis and Babis Chalios for their technical support in this research. The research leading to these results has received funding from the Research Foundation Flanders (FWO) and from the European Community's Seventh Framework Programme (FP7/20072013) under the People Programme (Marie Curie Actions), grant agreement no. 327744; the ENCORE Project (http://www.encore-project.eu), grant agreement no. 248647; the TEXT Project (http://www.project-text.eu/), grant agreement no. 261580; the SCoRPiO Project (http://www.scorpio-project.eu/), grant agreement no. 323872; the NovoSoft project (Marie Curie Actions), grant agreement no. 327744; and under the European Network of Excellence on High Performance and Embedded Architecture and Compilation (HiPEAC, http://www.hipeac.net), grant agreement no. 217068 and by the United Kingdom EPSRC GEMSCLAIM project, grant agreement no. EP/K017594/1.

Authors' addresses: H. Vandierendonck, G. Tzenakis, and D. S. Nikolopoulos, School of Electronics, Electrical Engineering and Computer Science, Queen's University Belfast; emails: h.vandierendonck@qub.ac.uk; gtzenakis01@qub.ac.uk; d.nikolopoulos@qub.ac.uk.

Permission to make digital or hard copies of part or all of this work for personal or classroom use is granted without fee provided that copies are not made or distributed for profit or commercial advantage and that copies show this notice on the first page or initial screen of a display along with the full citation. Copyrights for components of this work owned by others than ACM must be honored. Abstracting with credit is permitted. To copy otherwise, to republish, to post on servers, to redistribute to lists, or to use any component of this work in other works requires prior specific permission and/or a fee. Permissions may be requested from Publications Dept., ACM, Inc., 2 Penn Plaza, Suite 701, New York, NY 10121-0701 USA, fax +1 (212) 869-0481 or permissions@acm.org.

(c) 2013 ACM 1544-3566/2013/12-ART61 $\$ 15.00$

DOI: http://dx.doi.org/10.1145/2555289.2555316 


\section{ACM Reference Format:}

Vandierendonck, H., Tzenakis, G., and Nikolopoulos, D. S. 2013. Analysis of dependence tracking algorithms for task dataflow execution. ACM Trans. Architec. Code Optim. 10, 4, Article 61 (December 2013), 24 pages. DOI: http://dx.doi.org/10.1145/2555289.2555316

\section{INTRODUCTION}

The task dataflow model is gaining momentum to fill an important gap in the parallel programming landscape. This model aims to simplify parallel programming by annotating tasks in a program with the side effects that they incur on memory. To this end, the programmer or compiler determines the memory footprint of a task and annotates it with the potential side effect. Thus, it is made clear what memory a task may touch and whether it will be read or written. Next, the runtime system tracks dependences between tasks as they are spawned by analyzing overlap in memory footprints and $\mathrm{read} /$ write access modes. Using this information, it can schedule tasks with maximal parallelism while enforcing an execution order that yields results identical to serial execution.

The genericity of the task dataflow model has been demonstrated on scientific applications [Bosilca et al. 2010; Augonnet et al. 2010; Perez et al. 2008], but also on problems in bioinformatics [Agrawal et al. 2010] and H.264 video encoding [Alvanos et al. 2011] and decoding [Chi and Juurlink 2011]. The task dataflow model is applied to generic applications such as image analysis and cryptography [Jenista et al. 2011]. Moreover, it has been argued that task dataflow is an elegant way to express pipeline parallelism in general [Vandierendonck et al. 2011a].

Although the task dataflow model is very appealing in terms of programming simplicity, several recent works have reported significant overheads for dynamic dependence tracking. Best et al. [2011] show up to $40 \%$ overhead compared to the task time. Perez et al. [2010] report overheads per task spawn that are often around $100 \mu \mathrm{s}$ but can be as large as $6.8 \mathrm{~ms}$. Such overheads are clearly limiting the applicability and scalability of the task dataflow model. More importantly, the number of cores on a chip is quickly outgrowing the available memory bandwidth. As such, it is becoming increasingly important to strive for strong scaling [Dongarra et al. 2011]. Strong scaling occurs when a parallel program is executed on an increasing number of threads while the problem size is kept constant. Additional parallelism can be found in the program by performing less work per task, but this work is soon to be overshadowed by the sizable overheads of the runtime systems cited earlier.

This article contributes to task dataflow scheduling by presenting and analyzing techniques for dynamic dependence tracking that have overheads as low as $0.20 \mu \mathrm{s}$ per task spawn (about 400 cycles on our evaluation system) and are scalable to task graphs containing more than 1 million outstanding tasks. We arrive at this stateof-the-art performance by carefully analyzing the algorithms for dynamic task graph management and by presenting a series of improvements on the basic algorithms.

The main contribution of this article is to explicitly formulate and evaluate algorithms and accompanying data structures for dynamic dependence tracking. The literature is gravely failing to formulate the algorithms used. This is an important shortcoming in the literature, as we have found that naive implementations (including our first attempts, which are not reported here) perform orders of magnitude worse than the highly tuned algorithms presented in this article.

This paper is structured as follows. Section 2 presents a task dataflow programming model that provides the typical annotations one would expect to see in these models: input, output, in/out, commutative in/out, and reductions. Section 3 presents the execution model and analyzes the structure of the task graphs that may be built 
during execution. We identify two key concepts that characterize task graphs: versions, capturing renaming of arguments, and generations, capturing sets of parallel tasks.

Based on our analysis of task graphs, we present and analyze four schemes for dynamic task graph management in Section 4. The baseline scheme represents the task graph as a graph. An obvious approach, we show that the scheme is subject to runtime overheads of $O(N)$ per task, where $N$ is the number of tasks in the task graph. Our second scheme exploits the structure of the task graph and represents it as a hypergraph, where edges connect sets of nodes instead of a pair of nodes. This representation is much more economical and scales well to large task graphs and to large numbers of task arguments. Our third scheme builds on observations made on the hypergraph scheme and restyles the hypergraph to a list, using specific properties of task graphs. This reorganization yields the same performance scalability but reduces the constant in the performance equation. Besides these edge-centric schemes, we also consider an edgeless tickets scheme that synchronizes tasks using integers [Vandierendonck et al. 2011b]. Our experimental evaluation in Section 5 shows that despite our efforts to optimize the edge-centric schemes, edge-centric schemes cannot match the performance of the tickets scheme in the common cases.

Next, Section 6 discusses related work, and Section 7 concludes this article.

\section{PROGRAMMING MODEL}

We assume a task-based programming model that extends the Cilk language with dataflow annotations and execution [Vandierendonck et al. 2011a, 2011b]. In this language, the spawn keyword is inserted before a function call to indicate that the call may proceed in parallel with the continuation of the calling procedure. The sync keyword indicates that the execution of the procedure should be delayed until all spawned procedures have finished execution. Spawned functions may have arguments that are annotated with dataflow annotations. These annotations restrict the parallelism between spawned functions.

\subsection{Objects}

Objects are special program variables that can be used in task dataflow. Annotated task arguments can only accept objects as arguments (not constants or generic variable types). An object is an undivisible piece of memory for the purpose of dependence tracking. We assume that all objects are strictly nonoverlapping.

An object may be renamed, which means that its address is changed by the runtime system. The runtime system performs renaming to increase parallelism. The runtime system also makes sure that latent pointers to renamed objects are properly translated to the appropriate version of the object before accessing memory.

The runtime system associates metadata to each object (e.g. to perform dependence analysis and to recover its most recent version after renaming). The runtime system stores this metadata side by side with the object in order to speedup the retrieval of metadata.

\subsection{Memory Usage Annotations}

The arguments of spawned procedures may be annotated with memory usage information - that is, how the argument is accessed by the task. The memory usage may be input, output, input/output, commutative in/out, or reduction. An input argument is read but not written to. An output argument is written and may be read, but it is always written before it is read. Consequently, its value upon initiation of the task is irrelevant. An input/output argument (or in/out for short) may be read and written, and it may be read before it is written. 
A commutative in/out annotation extends the in/out semantics with the notion that consecutively spawned tasks may be executed in any order, although not at the same time (mutual exclusion). Naturally, reordering is subject to the absence of other intertask dependences.

A reduction is defined as a mathematical group consisting of an associative and commutative operator, together with a unit. ${ }^{1}$ The runtime system automatically provides private storage for reduction arguments in case multiple reductions on the same variable are executing concurrently. The runtime system also reuses the private storage in order to improve memory locality and to minimize applications of the reduction operator. When the value of a reduced variable is consumed by a task with a nonreduction annotation, then all private copies are automatically reduced to a single value. This reduction is performed serially for scalar reductions, and it is performed using a parallel tree reduction for nonscalar (e.g., matrix) reductions.

We stipulate that all arguments passed to a task are unique objects. This is to avoid circular dependences of a task on itself.

\section{EXECUTION MODEL}

The execution model of a task dataflow language assumes that a sequential thread of execution steps through the program and, in the process, encounters a sequence of tasks. This sequence, together with the memory usage annotations, defines dependences between tasks. A dependence states that a pair of tasks must execute in the order that they were spawned. These tasks are added one by one to the task graph, where nodes represent dynamic task instances and edges represent task dependences.

The task graph is a directed acyclic graph (DAG) because tasks can only depend on tasks that appear before them in (serial) program order. At any moment, the roots of the DAG are tasks that are either executing or are ready to execute. We call the list of root tasks that are ready to execute the ready list. It provides direct access to the ready tasks when one is needed.

\subsection{Task Graph Operations: Issue and Release}

The two main bookkeeping operations on a task are issue and release. Issue occurs when the task is spawned and implies that the task is linked to all tasks in the DAG on which it directly depends. If the task is a root of the DAG (no edges inserted for the task), then the runtime system may continue with the execution of the task; otherwise, it executes the continuation of the parent.

Release happens when the task has finished execution. At this moment, the task is removed from the DAG and from the ready list, and all of its dependents that become a root of the DAG are pushed on the ready list. At this moment, the runtime system selects a task for execution, which is either the parent procedure or a task on the ready list.

Note that the runtime system may be built such that multiple independent task graphs are operational for the same program, typically one task graph per procedure instance [Jenista et al. 2011; Vandierendonck et al. 2011b]. As such, issue operations on the same task graph never occur concurrently, as they are performed by the thread executing the function corresponding to that task graph. On the other hand, multiple release operations may occur concurrently, and they may occur concurrently with an issue operation.

\footnotetext{
${ }^{1}$ Cilk++ supports reductions using noncommutative operators [Frigo et al. 2009]. Our system is built on Cilk; thus, we assume that such noncommutative reductions are present. These do not require the dataflow annotations. We provide the commutative reduction annotation for increased performance because it requires fewer reduction operations.
} 
Table I. Dependences Arising Between Two Tasks Operating on a Common Argument

\begin{tabular}{|c|c|c|c|c|c|c|}
\hline & & \multicolumn{5}{|c|}{ First task } \\
\hline & & 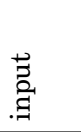 & 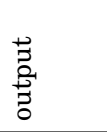 & 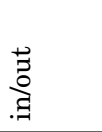 & 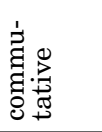 & 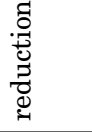 \\
\hline \multirow{5}{*}{ 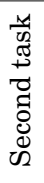 } & \multirow{5}{*}{$\begin{array}{l}\text { input } \\
\text { output } \\
\text { in/out } \\
\text { commutative } \\
\text { reduction }\end{array}$} & none & true & true & true & true \\
\hline & & anti & output & output & output & output \\
\hline & & anti & true & true & true & true \\
\hline & & anti & true & true & none $^{(x)}$ & true \\
\hline & & anti & true & true & true & none $^{(r)}$ \\
\hline
\end{tabular}
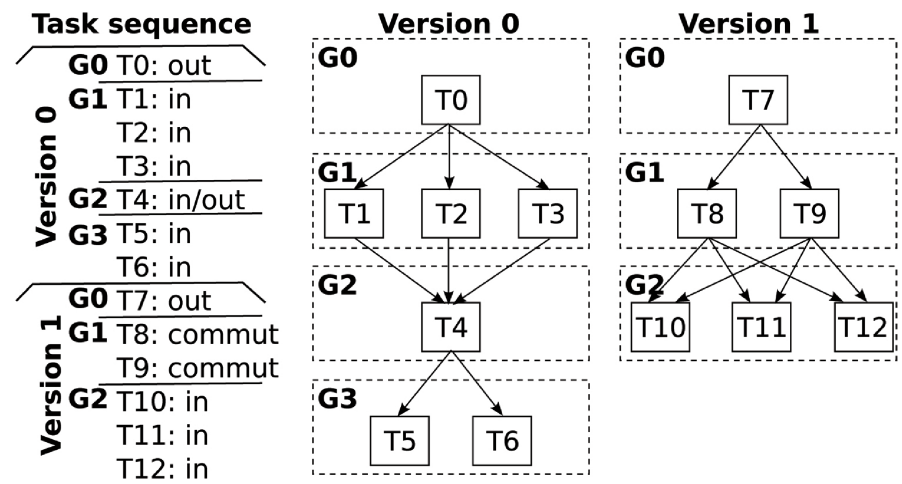

Fig. 1. A task graph structured in versions and generations. All tasks access a single object.

\subsection{The Structure of a Task Graph}

Tasks are ordered by true, anti-, and output dependences [Hennessy and Patterson 2003]. Table I shows the dependences between usage annotations. The entries "none $e^{(x)}$ "

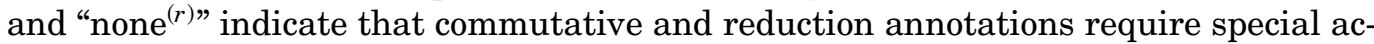
tions to ensure mutual exclusion and managing private copies, respectively. We consider these actions as separate steps from dependence tracking; thus, for the purpose of this article, we will treat these entries as meaning absence of dependence.

In this article, we will reduce the overhead of dynamic task graph management by exploiting properties that follow from Table I. Here, we assume that the tasks have only a single argument, an assumption that we will make throughout this article for reasons of simplicity. We claim that the presented reasoning applies equally well when aggregating our findings over multiple task arguments.

First, we notice that tasks with an output annotation may be easily renamed. Renaming eliminates anti- and output dependences by mapping an object to different storage space. We say that a new version of the object is created. A new version introduces new data storage for the object and has fresh metadata for dependence tracking that is initialized to an "unused" state. As such, distinct versions of the same object behave as if they were entirely distinct objects for the purpose of dependence tracking.

Figure 1 illustrates versions by means of a sequence of tasks in program order and the resulting task graph. Task T7 with output usage gives rise to renaming and the creation of version 1 . Tasks younger than T7 operate on version 1 , whereas older tasks operate on version 0 .

Second, we find that parallelism between tasks exists only between tasks with the same annotation ("none" occurs only on the diagonal of Table I). Distinct annotations imply dependences that must be enforced. 
We exploit this property by introducing the notion of generation of an object. A generation captures a series of successively spawned tasks that apply the same annotation to the object. An exception is the output and in/out annotations, where only one task is allowed per generation. Generations are ordered from old to young, where the oldest generation contains tasks that spawned first.

Generations give us the following semantics for parallel execution of the program:

Serialization between generations: generations must execute in program order-that is, all tasks from the oldest generation must have finished before tasks from the next generation may issue.

Parallelism within generations: the tasks within a generation may issue in any order.

Figure 1 illustrates the construction of generations: tasks in the same generation are independent; those in distinct generations are dependent.

Generations are a property of objects, so different objects may step through generations at a different rate. Real task graphs have multiple objects and are more complex than depicted in Figure 1, although we can always identify the structure presented here for each object in the program.

\subsection{Renaming}

We demonstrate that renaming of output annotation allows us to further optimize the task graph scheme by specializing code paths. This optimization performs renaming on output annotation, although not on in/out. If, however, for some reason ${ }^{2}$ that renaming is not possible, then the output annotation is modified to an in/out annotation. This retains correctness (an antidependence substitutes for an output dependence) but implies that we can always assume absence of older tasks accessing an object with output usage. We show in the evaluation that this improves the code paths measurably.

\section{TASK GRAPH MANAGEMENT}

Preliminaries. A task is stored in two separately allocated pieces of memory: the task structure and space for arguments and tags. The task structure is a fixed-size structure holding all task metadata, function pointer to call, pointer to the argument/tags block, and so forth. The argument/tags part stores the arguments as well as a variable amount of per-argument tag space. This tag space can hold any metadata specific to a scheme.

\subsection{The Graph Scheme}

The first task graph scheme represents the task graph as a graph by explicitly linking every task with all its direct dependents. As such, the task data structure is extended to contain a list of dependent tasks (deps), which is basically a list of outgoing edges from the task. The tag space is unused.

Figure 2 lists the issue and release actions for the graph scheme. The arguments to these actions are the object metadata and tags for the task argument, the argument annotation, and the task data structure.

Upon task issue, we know that the new task is either added to the youngest generation or that a new generation is created and is inserted there. In addition, the new task must be linked to all tasks in the previous generation. To accomplish this, it suffices that this scheme stores the two most recent generations of an object in a directly

\footnotetext{
${ }^{2}$ SMPSS uses a fixed-size buffer to allocate renamed storage from Barcelona Supercomputing Center [2008]. Renaming is turned off when all rename storage has been used. In our runtime system, the programmer can prohibit renaming of specific objects.
} 
1 issue (metadata md, tag_struct tags, annotation a, task_struct task)

2 md.lock()

3 // Start new generation if annotations mismatch

4 if $\mathrm{a}=$ in/out or md.annot[md.cur_generation] != a then

5 md.cur_generation : $=1$ - md.cur_generation

$6 \quad$ md.tasks[md.cur_generation].clear()

7 md.annot[md.cur_generation] := a

8 endif

$9 \quad$ /l Add task to current generation

10 md.tasks[md.cur_generation].push_back(task )

11 // Link task with tasks in previous generation

12 for task_struct prev_task in md.tasks[1-md.cur_generation] do

13 prev_task. $\operatorname{lock}()$

14 ++task.incoming_count // atomic add

15 prev_task.deps.push_back(task )

16 prev_task.unlock()

17 done

18 md.unlock()

19 release (metadata md, tag_struct tags, annotation a, task_struct task)

20 // Remove the task from the generation list that contains it

21 // (if it is in any list). Avoid scanning lists where possible.

22 md.lock()

23 if $\mathrm{md}$.annot[0] $=\mathrm{a}$ then

24 md.tasks[0].delete_task( task )

25 endif

26 if $\mathrm{md}$.annot[1] $=\mathrm{a}$ then

27 md.tasks[1].delete_task (task )

28 endif

29 md.unlock()

// Wakeup dependent tasks - this is executed once per task task.lock()

for task_struct dep_task in task.deps do

if - -dep_task.incoming_count $=0$ then $/ /$ atomic substract endif ready_list .push_back(dep_task )

\section{done}

task.unlock()

Fig. 2. Actions on a task graph represented as a graph. Note that task issue for output dependences uses specialized code where the tasks and annot fields are updated unconditionally and the linking step is skipped.

accessible way (when adding a task to the youngest generation, we must also know the previous generation to insert the links). It models the two most recent generations with a list of tasks and the corresponding annotation.

When releasing a task, it is removed from the lists of tasks from each of the last two generations (lines 23-28). There is no guarantee that the task is stored on any of those lists, as it may belong to even older generations.

When all arguments have been released, we walk the list of dependent tasks (line 33) and decrement their incoming edge counter. If this counter drops to zero, the task becomes ready to execute and is added to the ready list.

The graph scheme shows inefficiency in a number of cases. Assume that a generation of $M$ tasks is followed by a generation of $N$ tasks. Issuing a task in the first generation is constant-time, but issuing a task in the second generation takes $O(M)$ time to insert outgoing edges in each task in the first generation. Thus, task issue takes $O(M N)$ steps for $M+N$ tasks. 
More importantly, releasing a task in the first generation takes $O(M+N)$ time, as the previous generation list must be traversed to delete the task $(O(M))$ and because all outgoing edges must again be traversed $(O(N))$. Releasing a task in the second generation takes $O(N)$ time to delete the task from the generation lists. Thus, release takes $O\left((M+N)^{2}\right)$ timesteps for $M+N$ tasks. Both issue and release introduce a superlinear overhead when $M>1$ or $N>1$.

It is important to keep in mind that many research works adopt the graph scheme or a variation of it [Barcelona Supercomputing Center 2008; Augonnet et al. 2010; Agrawal et al. 2010] that is subject to the same flaws.

4.1.1. Optimization: Embedding Lists in Tags Storage. Task release time is influenced strongly by the deletion of a task from the "cur" and "next" generation lists. The graph scheme with embedded lists provides a $O(1)$ method to delete a task from the generation lists.

Assuming a circular doubly linked list implementation, we provide direct access to the linked list node corresponding to a particular task. Note that a task is inserted on one generation list for every task argument. As such, we provision space for a doubly linked list node in the tags space of the task, one node for each argument. The location of this node in memory is directly inferrable from its position in the argument list.

The list node contains previous/next link fields and a pointer to the task. It is initialized during issue (Figure 2, lines 6 and 10). Deleting a task from the list (lines 23-28) now takes $O(1)$ steps as the linked list must no longer be traversed to find the node.

Reconsidering the example with a generation of $M$ tasks followed by a generation of $N$ tasks, we calculate that task release now takes $O(M N)$ time for $M+N$ tasks. There is still a superlinear overhead when $M>1$ and $N>1$. Clearly not a solved problem, but an improvement over the graph scheme.

\subsection{The Hypergraph Scheme}

Performance anomalies disappear when we exploit the generational structure of the task graph. Namely, all tasks in one generation have an outgoing edge to the tasks in the next younger generation. These edges between tasks can be summarized by edges between generations. Such a construction is a directed hypergraph [Berge 1973], hence the name of this scheme.

The hypergraph scheme uses an explicit data structure to represent a generation. This data structure contains a list of tasks in the generation, a count of the number of tasks, and a pointer to the next younger generation. The generation data structure also contains a mutex variable to control concurrent accesses.

The metadata for an object now consists of a pointer to the current and previous generations of that object. Older generations may exist but are not referenced from the object metadata. The object metadata does not need mutually exclusive access, because it is only accessed by the thread that issues tasks. Generations are protected by a lock, and the locking order is from older to younger generations.

Figure 3 shows the issue and release algorithms. Where the graph schemes (Figure 2) store two generations and reuse this storage, the hypergraph scheme dynamically allocates new generations as they are created (line 5). Note that the incoming edge count for a task is incremented by one per incoming hyperedge (line 13), reducing the bookkeeping overhead.

This scheme uses the tag space to store a pointer to the generation of each argument that is used by this task (line 26).

Again, issue for an output annotation has a specialized code path that builds on the knowledge that both the previous and the current generation are empty. The only 
1 issue (metadata md, tag_struct tags, annotation a, task_struct task)

2 if ( $a=$ in/out or md.cur.annot ! $=a)$ and md.cur.num_tasks $>0$

3 then

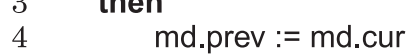

5 md.cur := new generation( annot :=a, next := null )

6 md.prev.next := md.cur

else

endif

md.cur.annot := a

if $\mathrm{md}$.prev != null then

md.prev.lock()

md.cur.lock()

task. incoming_count++ // atomic add

if md.prev.num_tasks $>0$ then

endif md.cur.tasks.push_back(task )

++ md.cur.num_tasks

md.cur.unlock()

else

md.prev.unlock()

// Don't need to build the list, it will not be traversed

md.cur.lock()

++ md.cur.num_tasks

endif

md.cur.unlock()

tags.gen := md.cur

release (metadata $\mathrm{md}$, tag_struct tags, annotation a, task_struct task)

tags.gen.lock()

- - tags.gen.num_tasks

I/ If there is a next generation, then wakeup all those tasks

if tags.gen.num_tasks $=0$ and tags.gen.next then

tags.gen.next.lock ()

for task_struct $t$ in tags.gen.next.tasks do

if -- t.incoming_count $=0$ then $/ /$ atomic substract

endif

ready_list .push_back(t )

done

// We do not need the list from now on

tags.gen.next.tasks.clear ()

endif

tags.gen.next.unlock()

tags.gen.unlock()

Fig. 3. Issue and release for the hypergraph.

necessary actions are to set the annotation (line 8) and the task count (line 23) of the current generation.

During task release, we decrement the number of tasks in the generation. When the currently oldest generation becomes empty, then we try to wake up each of the tasks in the next younger generation (lines 33-37). The scheme does not investigate the next younger generation as long as the oldest generation contains tasks. As such, it traverses the list of tasks in a generation only once per generation, as opposed to once per task in the graph scheme.

Furthermore, by careful structuring of the code, we obviate the need to delete a task from its generation during release. In fact, during release, we erase the list of tasks at once when a generation is woken up (line 39). After this, the list of tasks is no 
longer needed, neither for issue nor release. Corner cases require that a generation's task list is not updated when the previous generation is empty (line 14) or nonexistent (line 20).

This design choice has a huge consequence on the time complexity of the issue and release steps. If we reconsider the example of the task graph with generations of $M$ and $N$ tasks, respectively, we find that the time complexity of issue and release is now $O(1)$ when amortized over all tasks in the task graph. Consequently, embedding the storage of linked list nodes in the tag space of the task arguments now does not improve the performance scalability of the scheme, although it will have some benefit due to a reduction in memory allocations.

\subsection{The List Scheme}

Optimizations to the generational scheme have demonstrated several principles that allow an organization around lists of tasks rather than generations. This results in a simpler organization of the task graph but maintains the parallelism.

The algorithm is presented in Figure 4. The key insights behind the list scheme are as follows:

(1) At any one time, the task graph is operating on only two generations: the oldest generation, consisting of the tasks that are currently ready to execute (as far as this particular argument is concerned), and the youngest generation, where newly issued tasks are added.

(2) For the oldest generation, we only need to know how many tasks are still executing (Figure 4, line 23). When the last task in a generation has finished, we need to wake up tasks in the next generation (line 33).

(3) For the youngest generation, we only need to know the annotation of the tasks in order to switch to a new generation whenever the annotation changes or demands so (in case of in/out usage) (line 10).

(4) All tasks operating on an object may be kept on a single ordered list, provided that a marker is inserted on the last task in each generation. Task issue appends tasks to the tail of this list. During task release, when moving to a new generation, we traverse the task list until the end-of-generation marker is reached or otherwise the list runs empty. The task count in the oldest generation is updated with the number of tasks encountered.

(5) Sometimes, the youngest and oldest generations are the same. This special situation must be properly accounted for by updating the task count in the oldest generation during task issue when there is one generation in the task graph (line 23). It also requires resetting of the annotation in the youngest generation when the task graph runs empty (line 48).

Accesses to the task graph metadata in the list scheme must be protected by mutual exclusion as in the other edge-centric schemes. We have experimented with a version of the scheme with a single lock and one with two locks. In the first version, the lock protects both the youngest and oldest generations. In the second version, distinct locks are used to provide access to the youngest and oldest generations. However, both locks must be acquired when there are two or fewer generations. Overall, we found the single-lock variation more performant. We attribute this to the fact that our scheduler executes most often in a scenario where only part of the task graph is instantiated at a time.

\subsection{The Tickets Scheme}

The ticket scheme was originally described for a model with input, output, and in/out annotations [Vandierendonck et al. 2011a, 2011b]. In this article, we make a minor 
1 issue (metadata md, tag_struct tags, annotation a, task_struct task)

tags.task := task // tags are nodes in a linked list

tags. last_in_generation $:=$ false

tags.next := null

md.lock()

if md.num_gens $=0$ then

md.num_gens := 1

md.oldest_num_tasks := 1

md.youngest_annot:= a

else if $(a=$ in/out or md.youngest_annot != a ) and md.youngest_annot != empty then

md.youngest_annot := a

++ md.num_gens

++ task.incoming_count // atomic add

if md.tasks. tail != null then // append to linked list

md.tasks. tail . last_in_generation := true

md.tasks. tail . next := tags

else

endif

md.tasks.head := tags

md.tasks. tail $:=$ tags

else

if md.num_gens $=1$ then

++ task.incoming_count // atomic add

md.tasks. tail .next := tags // append to linked list

endif

md.tasks. tail $:=$ tags

endif

md.unlock()

release (metadata $\mathrm{md}$, tag_struct tags, annotation a, task_struct task)

md.lock()

if -- md.oldest_num_tasks $=0$ then

if md.tasks.head!= null then

do

tag_struct $t:=$ md.tasks.head

if - t.task.incoming_count $=0$ then // atomic substract

endif ready_list .push_back(t.task )

++ md.oldest_num_tasks

md.tasks.head := t.next

endif

while $\mathrm{t}$. last_in_generation = false and md.tasks.head != null

- -md.num_gens // generation moved to ready list

if $\mathrm{md}$.tasks.head $=$ null then

md.tasks. tail $:=$ null

if $\mathrm{md}$.num_gens $=0$ then

endif

md.youngest_annot := empty

endif

endif

md.unlock()

Fig. 4. Issue and release for the scheme organized around a task list. 
extension to the scheme to include commutative in/out and reductions. We provide a slightly different exposition of the algorithm that is rooted in our terminology of generations.

The scheme resembles a ticket-based queuing system, such as that operated in a butcher's store. The ticket queuing system uses two counters: a global counter and a next counter. The next counter serves to serialize all clients: each client gets a successive value of the next counter, which is called a ticket. The global counter is incremented for every client served. At any time, the client to be served is the one whose ticket equals the global counter.

The ticket scheme that implements task graphs uses four ticket queues per version of an object referred to as R (input), W (output and in/out), C (commutative in/out), and $\mathrm{P}$ (reductions). We use different ticket queues per annotation because we need to synchronize between generations but not within generations. When a task issues, it increments the next counter in the queue that corresponds to its annotation, and it copies the next counter from each of the other queues (it takes three tickets). It becomes ready for execution when the global counters in the other queues match the corresponding tickets. This means that any task with a different annotation and that issued earlier must have finished execution before the task can execute. There is, however, no synchronization with other tasks with the same annotation, because we want these tasks to execute in parallel. ${ }^{3}$ After serving a task, the global counter in the queue that matches the task's annotation is incremented.

Tasks with in/out annotations must also serialize with other tasks with in/out annotation. We enforce this synchronization by making these tasks also wait on the $\mathrm{W}$ queue, such that they take/return tickets from the same queue on which they wait.

Figure 5 shows the issue and release operations under the ticket algorithm. The object metadata consists of eight counters (four ticket queues of two counters each). The tag space for an argument is used to store ticket values that must be waited on. As such, the tags for an input, commutative, and reduction annotation contain three values, the tags for an output annotation are empty, and the tags for an in/out annotation contain four values.

The issue and release actions must not execute in exclusion from related actions on the same versions, provided that every increment in the release operations is performed atomically. Increments in the issue operations need not be atomic, because the issue and release actions modify different variables and because the task graph is generated by a single thread of execution.

The ticket scheme has one peculiarity: as tasks do not contain pointers to the task that they may wake up, it is not possible to populate a ready list. Instead, the ticket scheme keeps all tasks in a pool and searches through this pool for ready tasks when a new task to execute is demanded [Vandierendonck et al. 2011b]. To this end, a ready check must be defined for every argument annotation. This ready check consists of comparing the appropriate global counters in the object metadata to the tickets stored in the tag space of an argument. The tasks in the pool are organized by their depth in the task graph in order to limit overhead when searching for a successor. The pool is organized as a resizable hash table with chaining where each chain stores the tasks with a particular depth.

\footnotetext{
${ }^{3}$ Note that tasks with the same annotation and that belong to different generations must be synchronized. This is accomplished by transitivity: there must be a generation in between with a different annotation that is synchronized with the other two generations.
} 


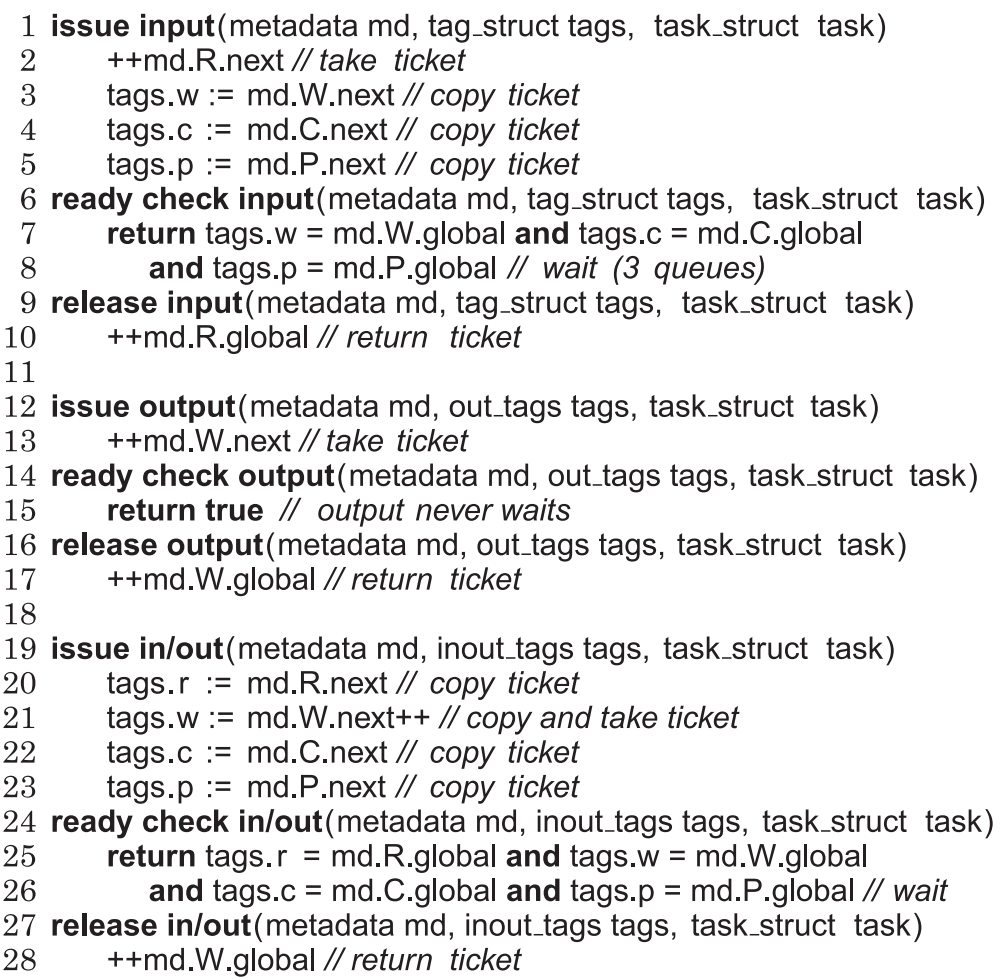

Fig. 5. Actions for the ticket scheme. The commutative in/out and reduction annotations are similar to the case of input annotation.

\section{EVALUATION AND ANALYSIS}

We evaluate the task graph schemes using microbenchmarks. The evaluation platform is a 48 -core AMD Opteron 6172 at $2.1 \mathrm{GHz}$. The codes are compiled using gec 4.7.3 at optimization level -O4.

We measure time in the microbenchmarks using the cpuid/rdtsc instruction sequence to measure delay in cycles. Although this instruction sequence disturbs execution time somewhat by serializing all instructions, it is an appropriate way to measure time delays on the order of 100 s of cycles. Moreover, we measure multiple occurrences of an event and present the average delay over those events to mitigate this source of imprecision.

The task graph schemes determine what tasks are ready to execute. The scheduler must choose which of these tasks to execute and on what core. These are two distinct problems. We assume a greedy scheduler in this work, such that the first task on the ready list is scheduled on the first core that becomes idle. The scheduler is a work-stealing task dataflow scheduler [Vandierendonck et al. 2011b] that implements thework-first principle, as in Frigo et al. [1998].

\subsection{Fast Execution Path: All Tasks Are Ready}

We constructed a microbenchmark that measures the fastest path in our system to spawn a task. It contains a single thread of execution that spawns and immediately executes a number of one-argument tasks. All tasks are passed the same object. Task issue operates on an empty task graph, and task release never wakes up dependent tasks. The microbenchmark bypasses queueing of tasks, as our system immediately 


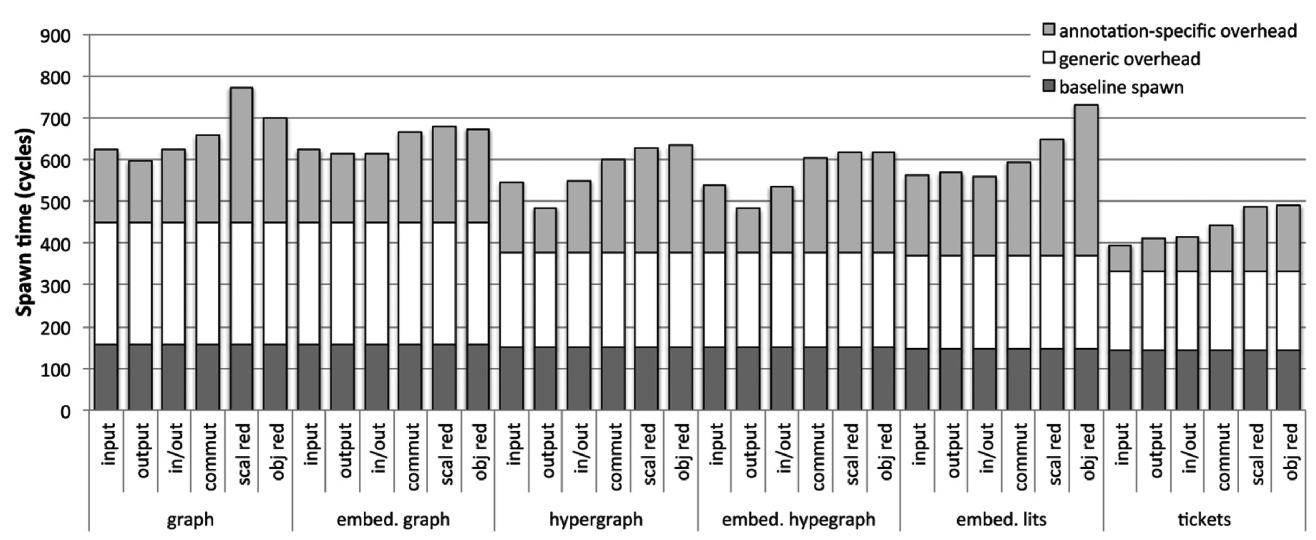

Fig. 6. Delay of fast execution path when spawning and immediately executing a task with one ready argument. The abbreviation "commut" stands for the commutative annotations, "scal red" is a reduction using serial accumulation of results, and "obj red"is a reduction using parallel tree reduction.

executes a task when it is ready at the moment of the spawn. This behavior is similar to Cilk's work-first principle [Frigo et al. 1998], which is implemented in our scheduler. Consequently, this fast execution path is an important and common behavior also for dependence tracking.

Figure 6 shows the delay of the fast execution path measured in processor clock cycles, averaged over 10 million spawns. We show results for the task graph schemes discussed in this article and all supported annotations. The spawn delays are broken down in three parts. The baseline spawn time equals the time to spawn and executes an empty task that has no arguments. The generic overhead is the overhead that is incurred due to task dependence tracking. These overheads are the largest in the graph schemes; they are smallest in the tickets scheme. Embedding generation lists hardly affects the delay of the fast execution path because the generation lists contain one task at most.

The annotation-specific overhead is the overhead that is introduced to track a particular dependence type on top of the generic infrastructure. Output dependences incur the least annotation-specific overhead, because task issue is optimized to the situation where no prior tasks are operating on the same object. The commutative in/out annotation incurs somewhat more overhead than the in/out annotation, because the runtime system implements exclusion by means of a mutex that is stored in the object metadata. The reduction annotations are also more expensive because of the management and reduction of private versions of the object.

Overall, the tickets scheme introduces the least generic overhead and the least annotation-specific overhead. The task spawn delay in the tickets scheme can be as low as 400 cycles or $0.20 \mu$ s on our evaluation machine.

\subsection{Slow Execution Path: All Tasks Require Wakeup}

The slow path for executing a task involves an initial ready check, issue, execute, and release. We measure the slow path with a microbenchmark that artificially inserts an active task in the metadata of an object and then spawns $N$ tasks that access the same object. These tasks have a variable annotation (output, in/out, etc.) but perform no work. Next, the microbenchmark releases the artificial task and then waits for all issued tasks to wake up and complete. This process is repeated several times to capture variance on the measurements. The microbenchmark uses one thread of execution to factor out multithreading overheads. 

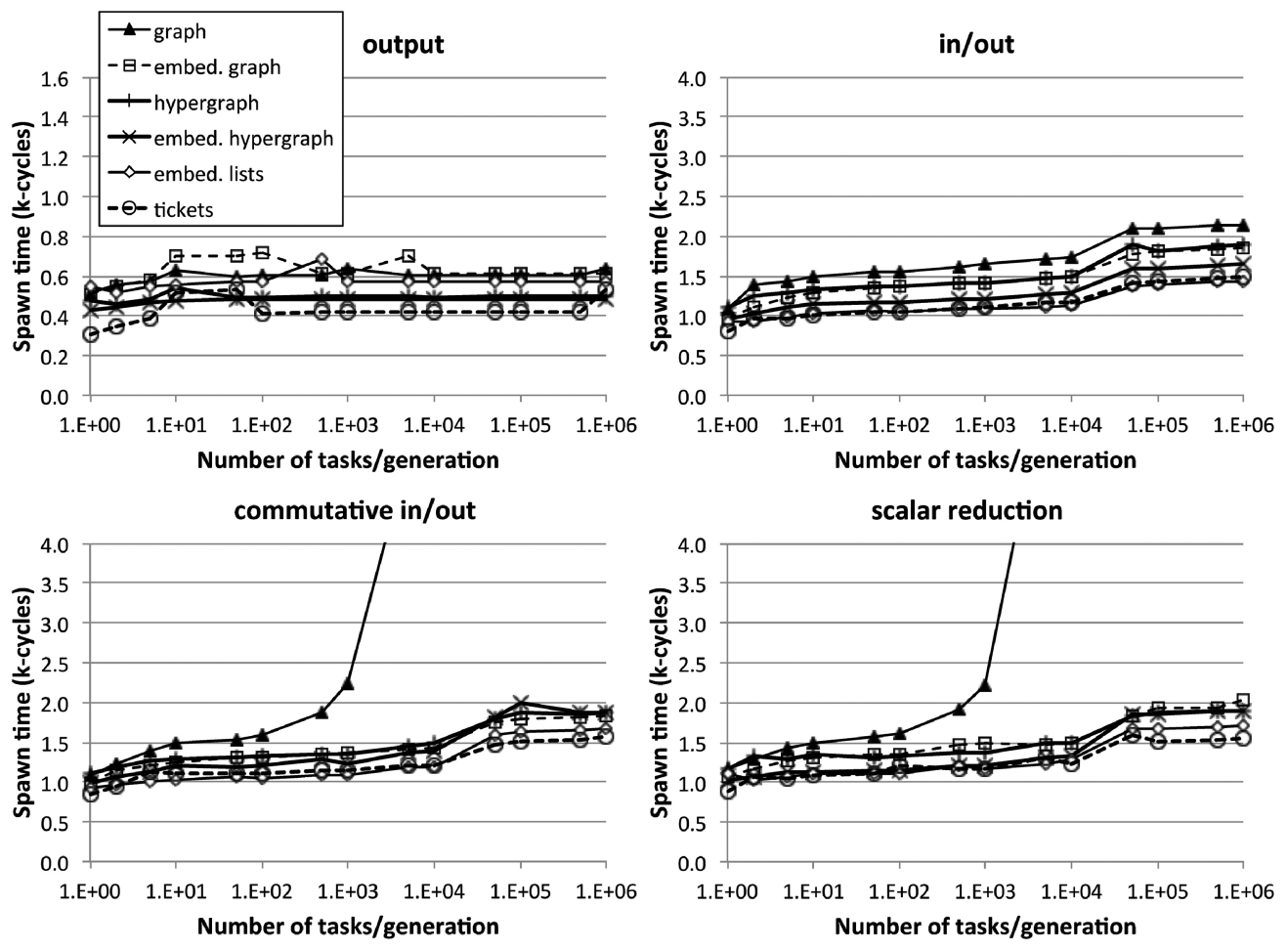

Fig. 7. Spawn time for the slow-path microbenchmark with two annotations.

Figure 7 shows the spawn overhead in this microbenchmark for a representative set of the annotations. Other annotations lead to similar results. The number of tasks in a generation is varied on the horizontal axis. The vertical axis shows the average delay per task spawn. These results confirm that the graph scheme introduces a super-linear overhead when $N>1$ (see Section 4.1). Moreover, the embedded graph scheme does not suffer superlinear overheads, because the first generation consists of just one task $(M=1)$. The hypergraph schemes have no scalability problems.

Note that $N=1$ for output and in/out annotations because a new generation is constructed for every task. Thus, for this microbenchmark, all graph scheme are scalable. In addition, renaming is applied for the output annotations, so the tasks are spawned at a speed close to the fast-path speed.

Three-Generation Variant of the Microbenchmark. We also constructed a microbenchmark with three generations: one in/out task that is artificially delayed, a sequence (generation) of $N$ tasks with input annotation, and a sequence of $N$ tasks with variable annotation. Figure 8 illustrates the shape of the task graph of this microbenchmark. Figure 9 shows the performance of the task graph schemes for a representative set of the annotations.

The graph scheme shows a scalability problem on output and in/out annotations when the number of tasks approaches 10,000. This is due to deleting the input tasks from the generation lists.

With the commutative in/out and scalar reduction annotations, the task graph is structured as two generations of $N$ tasks each. In this case, our analysis (Section 4.1.1) has already shown that the graph and embedded graph schemes are not scalable. 


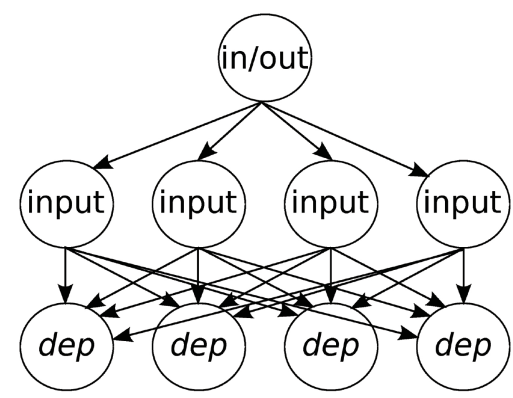

Fig. 8. Task graph constructed in the three-generation slow path microbenchmark for $N=4$.
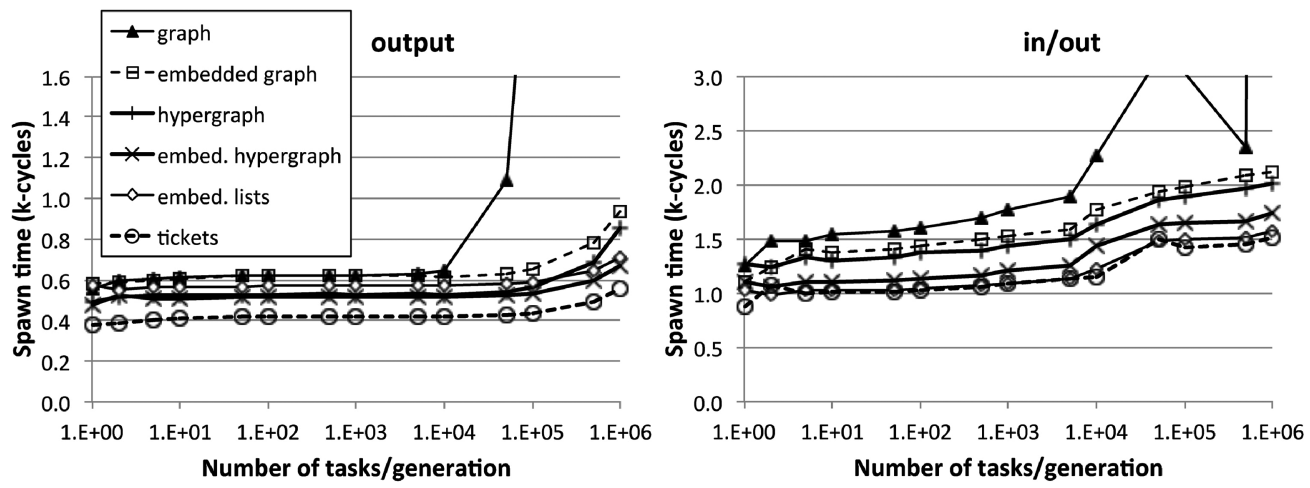

commutative in/out
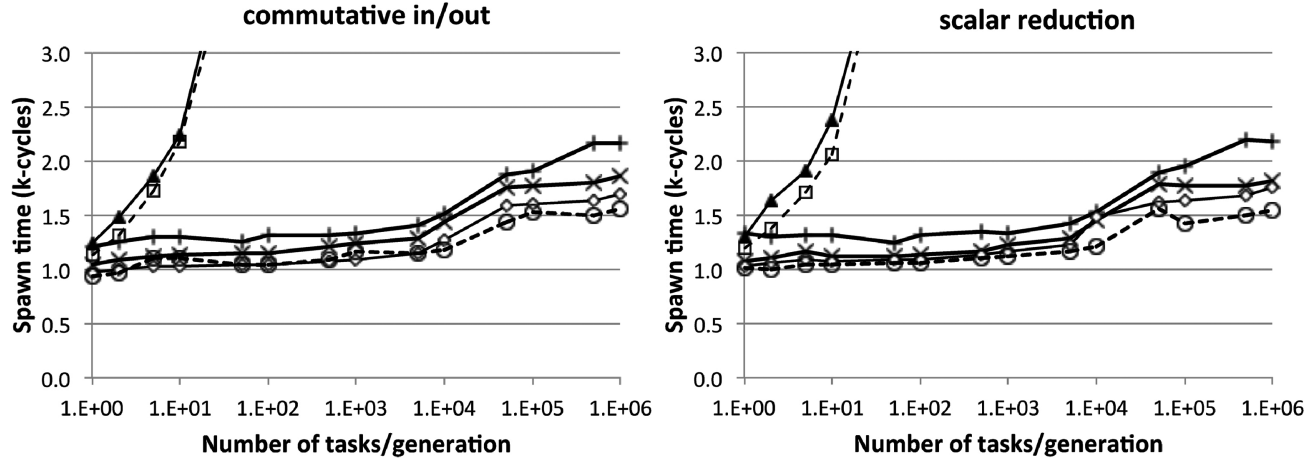

Fig. 9. Spawn time for the slow-path microbenchmark with three generations.

Overall, the tickets scheme introduces the least overhead. The hypergraph schemes also perform well and introduce up to around 30\% and 15\% slowdown over the tickets scheme for the hypergraph and embedded hypergraph schemes, respectively, when $N \leq 1,000$. The embedded lists scheme has an average overhead of $5 \%$ compared to the tickets scheme.

\subsection{Slow Execution Path: Work Stealing}

We measure the runtime system overhead in the presence of work stealing using a microbenchmark that issues several thousand tasks with an input annotation. We pass the same object to each task's argument. We execute this benchmark using 48 cores. Figure 10 shows the observed delay of a task spawn in the microbenchmark. 

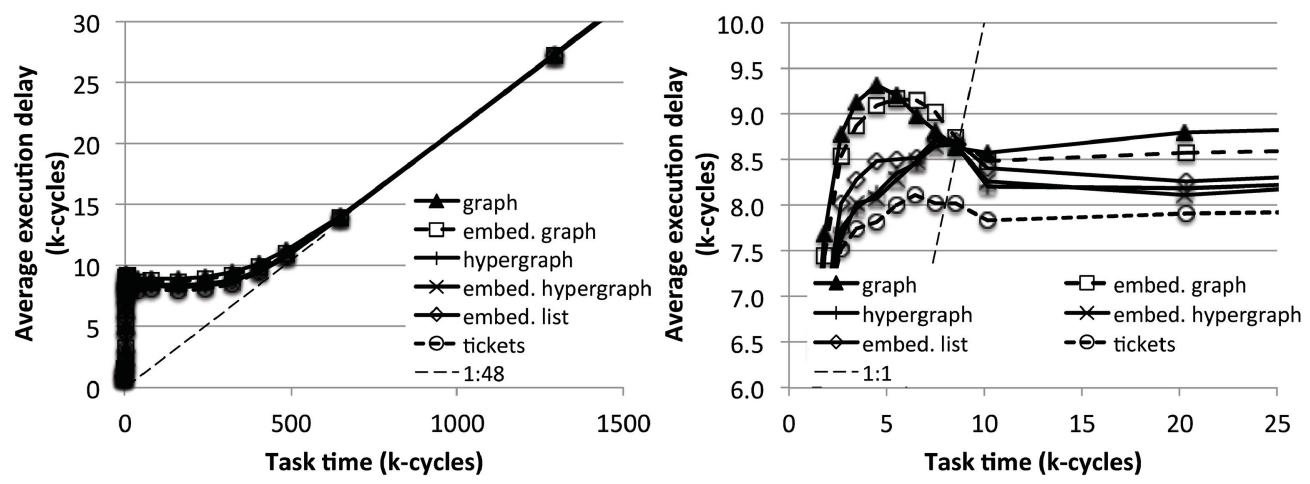

Fig. 10. The impact of task size on scalability when executing with 48 threads. The graph on the right zooms in on the graph on the left.

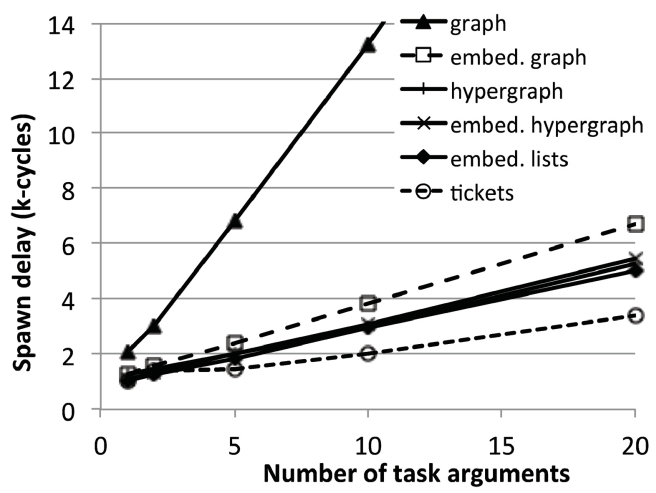

Fig. 11. Varying number of task arguments.

We vary the size of the tasks on the horizontal axis. In each case, the number of tasks is chosen such that the total execution time remains in the 1 to 10 s range.

The results show that the scheduler obtains linear scaling at a task size of about 190 to $230 \mu \mathrm{s}$ (around $400 \mathrm{~K}$ cycles). The task graph scheme used has little impact hereon. This is expected, as the task graph schemes show performance differences in the submicrosecond range.

The task graph scheme has a noticeable impact when the scheduler does not achieve linear scaling. In this case, the tickets scheme is 10 to $20 \%$ faster than the graph scheme and up to $8 \%$ faster than the embedded lists scheme. Note that the majority of the 8,000-cycle task execution delay measured here is due to the scheduler and the way it performs task stealing. This is not affected by the task graph scheme.

We also have performed this experiment when using up to twice as many objects as there are threads. Objects are accessed in round-robin manner such that subsequently spawned tasks operate on distinct objects. The results are similar to Figure 10, showing that the objects and the task graph data structures are not a point of contention.

\subsection{Number of Task Arguments}

Figure 11 shows the spawn delay of the slow-path microbenchmark where the number of arguments per task is varied. A different object is passed to each argument. The overhead of the edge-centric schemes becomes more pronounced as tasks have more 

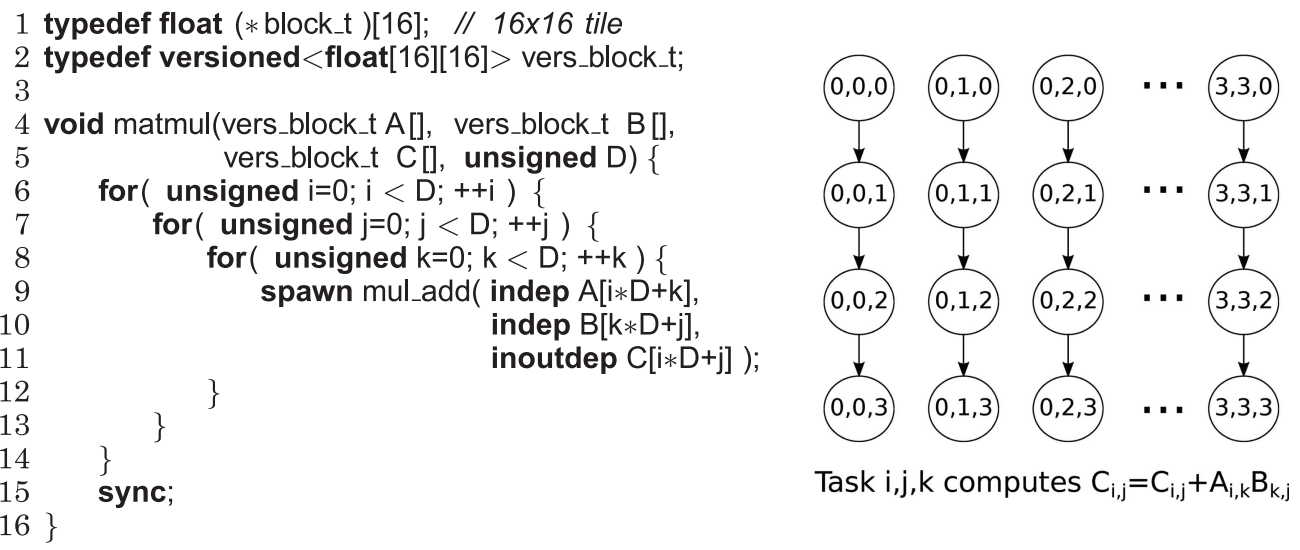

Fig. 12. Left: Task dataflow program for matrix multiply over $\mathrm{D} \times \mathrm{D}$ matrices of $16 \times 16$ blocks using an "ijk" loop order. Adapted from Vandierendonck et al. [2011b]. Right: The task graph of matrix multiply when $\mathrm{D}=4$.

arguments. At 20 arguments, the best edge-centric scheme is more than $30 \%$ slower than the tickets scheme. The graph scheme shows unacceptable slowdowns.

Although 20 arguments for a task seems high, it is not unreasonable because the programming model discourages the use of global variables. Global variables cannot participate in dataflow dependences. Our implementation of the PARSEC Blackscholes benchmark (e.g., it has a task with eight arguments, of which seven are annotated). Here, the tickets scheme already outperforms the other schemes by $25 \%$.

\subsection{Microbenchmarks with Realistic Task Graphs}

We evaluate the task graph schemes on microbenchmarks that exhibit the same task graph as real codes: matrix multiply and Cholesky factorization. The actual tasks in these microbenchmarks are configurable, as in the experiment shown in Section 5.3.

5.5.1. Task Graph: Matrix Multiply. The matrix multiply mimicked is a block matrix multiply where the result matrix is divided in $D \times D$ blocks of a fixed size, in this case $16 \times 16$ blocks (Figure 12). For matrices of $D \times D$ blocks, the program generates $D^{3}$ tasks, where disjoint groups of $D$ tasks update the same block of the $C$ matrix, which causes serialization of these tasks. Considering that there are $D^{2}$ distinct blocks in the $C$ matrix, the task graph shows $D^{2}$ strands of $D$ tasks each. In the experiments presented here, $D=128$. Due to the regularity of the task graph, matrix multiply is not a key motivator for task dataflow languages. This benchmark is, however, helpful for performance analysis.

Figure 13 shows the average task execution time for varying amounts of work per task and also for two loop orderings of matrix multiply. On the lefthand side, the "ijk" loop order is used. Here, the innermost $k$-loop computes the product for one block of the result matrix. Tasks are traversed one strand of the task graph at a time. Consequently, each task in the inner loop depends on the previous one, and the scheduler must scan ahead in the program to the next iteration of the $j$-loop to expose parallelism. Based on the properties of the scheduler, we can calculate that execution of this program uses $O\left(D^{2}\right)$ work stealing events (i.e., one per strand in the task graph). Note that work stealing is significantly more expensive than creating and initiating a pending task.

With the "ijk" loop order, the graph scheme shows a performance anomaly where tasks shorter than $150 k$-cycles incur increased delay. The tickets scheme performs worse than the other schemes. As the structure of the program forces construction of 

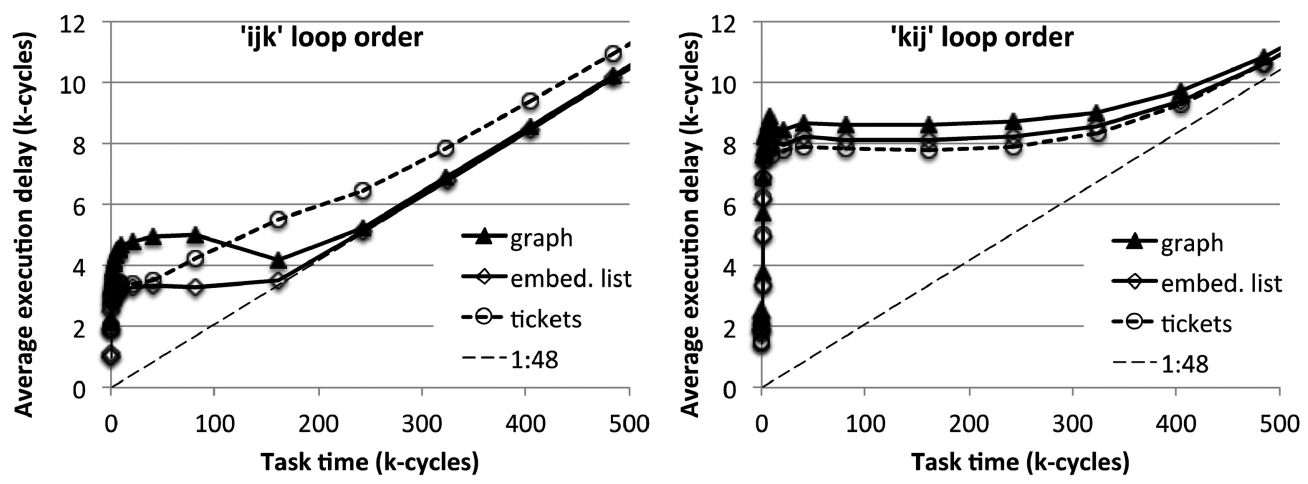

Fig. 13. Impact of the task graph schemes on the performance of blocked matrix multiply.

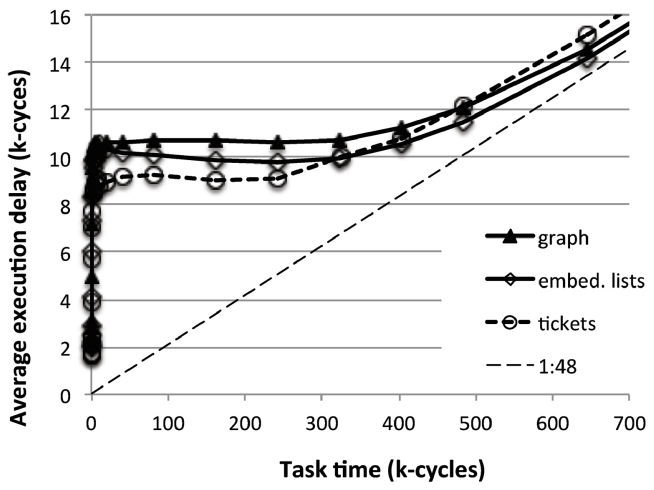

Fig. 14. Impact of the task graph schemes on the performance of the Cholesky factorization skeleton.

nearly the full task graph, additional pressure is put on the pool to locate the next ready task.

On the right-hand side in Figure 13, a "kij" loop order is used. This loop order launches one task for every block in the result matrix before launching the second task on the first block. As such, tasks are traversed by visiting each strand in the task graph in round-robin fashion, and the program has a great deal of readily exploitable parallelism. However, this task traversal order also implies that $O\left(D^{3}\right)$ work stealing events are used to execute the program, assuming that $D^{2} \gg P$, where $P$ is the number of processors.

The tickets scheme outperforms the other task graph schemes, typically by about 400 cycles or $3.5 \%$ of the total task delay. Note that the average task delay is in the best case about 4,600 cycles larger for "kij" loop order than for the "ijk" loop order. This is a side effect of the design of the scheduler and the cost of work stealing, in combination with an increase in work stealing events by $O(D)$.

We have not shown the embedded graph and hypergraph schemes, as they perform nearly as well as the embedded lists scheme.

5.5.2. Task Graph: Cholesky Factorization. Figure 14 shows the performance of the task graph schemes on the task graph for Cholesky factorization of a matrix divided in $128 \times 128$ blocks. The results confirm previous findings: the tickets scheme is inherently faster than the edge-centric task graph schemes at very small task sizes. It outperforms the embedded lists scheme by 10 to $15 \%$ for task times of $250 \mathrm{k}$ cycles and less. However, 

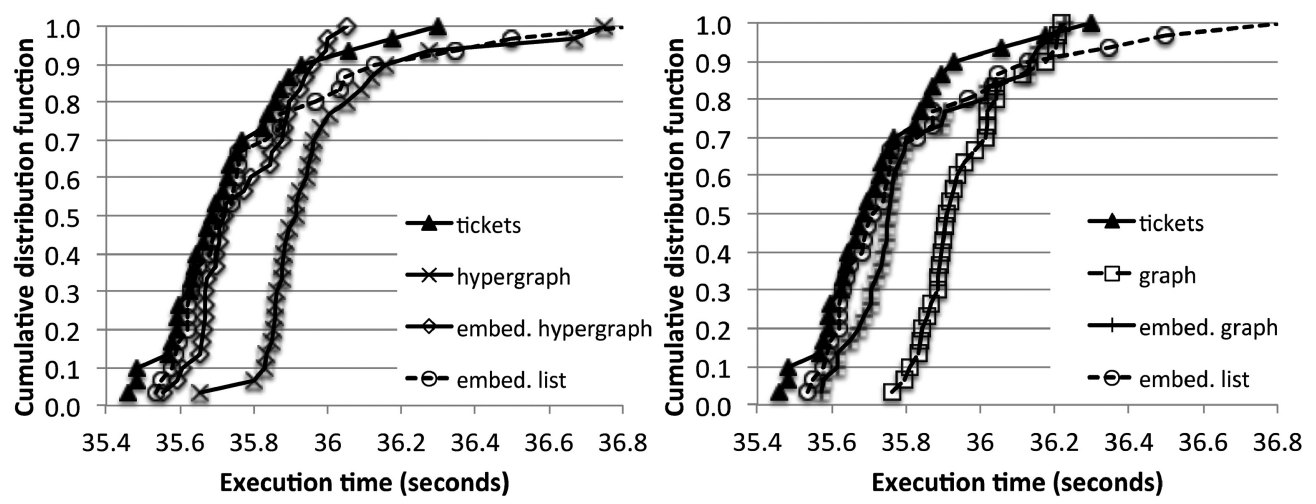

Fig. 15. Cumulative distribution function of the execution times of ferret over 30 executions, when using each of the dependence tracking schemes. The left and right graph show different subsets of the results. The graph and hypergraph schemes incur similar execution times, causing their curves (shown in distinct graphs for clarity) to overlap.

Cholesky factorization has a complex task graph, and the pool fails to locate the next ready task sufficiently fast. For $500 \mathrm{k}$ cycle tasks, the tickets scheme is $3 \%$ slower than the task graph schemes. The performance of all schemes converges as task time increases.

\subsection{Benchmarks}

We study the impact of performance on two benchmarks with higher complexity, namely ferret and dedup, taken from the PARSEC benchmark suite [Bienia 2011]. We have analyzed the performance of these benchmarks using our runtime system in other work [Vandierendonck et al. 2013], where we have also proposed new programming language concepts to accelerate these applications and improve their programmability. In this work, we evaluate the baseline versions using input, output, and in/out annotations.

5.6.1. Ferret. The parallelism exploited in ferret consists of a five-stage pipeline where images are processed and compared in successive steps. Ferret's performance scales nearly linearly with an increasing number of cores [Vandierendonck et al. 2013]. Ferret has fine-grain tasks, which causes differences between the dependence tracking schemes to show up in the overall execution time. Figure 15 shows the cumulative distribution function of overall execution time of ferret on 48 cores over 30 runs, when executing the native input set supplied with PARSEC.

The tickets scheme results in lower execution times than the other schemes, although the difference with the lists scheme is negligible. The main result of this experiment is that the graph and hypergraph schemes are clearly slower than their embedded graph and embedded hypergraph counterparts. Given the overlap of measured execution times between different schemes, we validate this result statistically.

We use the two-sided Wilcoxon rank sum test [Conover and Iman 1981], a nonparametric test, to establish equality of means. We test the null hypothesis that two schemes yield the same mean execution time with a significance level of $5 \%$. The difference between the tickets scheme and the graph scheme is statistically significant ( $\mu=35.74 \mathrm{~s}$ and $\sigma=0.19 \mathrm{~s}$ for tickets vs. $\mu=35.96 \mathrm{~s}$ and $\sigma=0.13 \mathrm{~s}$ for the graph scheme) with a Wilcoxon value of $W(30)=132$ and $p=2.58 e-6$, with failure to accept the null hypothesis. A similar statistically significant difference can be measured between the tickets and hypergraph schemes. 
Table II. Range of Execution Times Measured for 30 Runs of Dedup When Executing on 16 Cores, Using Each of the Dependence Tracking Schemes Threads are packed and pinned on the cores of three processors.

\begin{tabular}{|l|c|c|c|c|}
\hline & mean (s) & std.-dev. (s) & $\min (\mathrm{s})$ & $\max (\mathrm{s})$ \\
\hline \hline tickets & 6.60 & 0.27 & 6.11 & 7.23 \\
\hline graph & 6.65 & 0.23 & 6.33 & 7.23 \\
\hline embedded graph & 6.71 & 0.29 & 6.13 & 7.45 \\
\hline hypergraph & 6.72 & 0.34 & 6.25 & 7.75 \\
\hline embedded hypergraph & 6.60 & 0.42 & 5.97 & 7.47 \\
\hline embedded lists & 7.22 & 1.42 & 6.24 & 10.85 \\
\hline
\end{tabular}

The tickets scheme does not differ from the remaining schemes in a statistically significant way. For example, compared to the embedded graph scheme $(\mu=35.82 \mathrm{~s}$, $\sigma=0.19 s)$, we find $W(30)=321$ and $p=5.75 \%$, so we cannot reject the null hypothesis.

In summary, we find that the graph and hypergraph schemes are slower than the other schemes with statistical significance, but there is no statistically significant difference in average performance between the tickets scheme and the embedded variants. These results demonstrate that the embedding optimization is crucial, as it minimizes the operations in the dependence tracking scheme, especially those involving extra memory allocations and deallocations.

5.6.2. Dedup. Dedup is an application with nested pipelines-that is, one pipeline stage in the outer pipeline is decomposed as another pipeline with finer-grain parallelism [Bienia 2011]. As such, dedup is an example of a program with nested task graphs. The version we test here is slightly different from the baseline version in Vandierendonck et al. [2013], as we have collapsed three stages in the inner pipeline (hash computation, hash table access, and compression), because this gives better performance.

The performance ranges of dedup are summarized in Table II when 16 cores are utilized. Dedup quickly runs out of parallelism. The measurements on 16 cores reflect a situation where threads are essentially idle and engaging in work stealing. We observe that the performance of dedup is independent of the dependence tracking scheme. Although some difference on the average execution time can be observed, these are much less than the standard deviations. As such, the Wilcoxon test does not validate these differences as statistically significant.

All schemes have fairly heavy tails, which is probably typical for programs engaging frequently in locking. Some executions with the embedded lists scheme are much slower (up to $73 \%$ larger than the shortest execution time). We do not have enough data to conclude whether this is an artifact of the implementation or whether it is typical for the embedded lists scheme, as it only shows up with this severity in dedup.

\section{RELATED WORK}

\subsection{Programming Model}

The literature presents many variations of the task dataflow model. Several articles use the basic model with input and output annotations [Agrawal et al. 2010; Gupta and Sohi 2011], supplemented with the in/out annotation [Chan et al. 2007]. CnC considers only inputs and outputs and calls them producers and consumers [Budimlić et al. 2010]. What SMPSS calls a reduction [Barcelona Supercomputing Center 2008] is in fact commutativity in our model, except that SMPSS requires the programmer to lock the reduction variable when it is accessed. The annotation is renamed to "concurrent" in OmpSs [Duran et al. 2011], the successor to SMPSS. In this work, it is the runtime that ensures exclusive access. This work also implements a real reduction annotation 
that automatically manages multiple copies of the reduction variable. In this respect, the annotation is as strong as a Cilk++ reducer [Frigo et al. 2009]. Cilk++ reducers allow noncommutative operators, but our system assumes commutativity for practical reasons.

\subsection{Task Graph Management}

Most articles that discuss runtime systems for task dataflow languages are vague about the implementation of the dependence tracking algorithms, with the underlying rationale being that there is no risk of scalability problems, or the assumption that the advantages of exposing additional parallelism will always outweigh its cost. This article has shown that these assumptions are false: there is a risk of lack of scalability if the task dependence tracking is implemented using a graph representation of the task graph.

We were able to determine through analysis of source code that some systems construct a graph that explicitly contains edges for every pair of dependent tasks [Barcelona Supercomputing Center 2008; Augonnet et al. 2010; Agrawal et al. 2010], as in the graph scheme. As such, they are prone to the pitfalls of this scheme.

SuperMatrix implements Tomasulo's algorithm in software [Chan et al. 2007]. The presented scheme is limited to one output or one in/out argument per task and does not support commutativity or reductions. As such, the programmability of the system is reduced. More importantly, Tomasulo's algorithm as designed with a hardware implementation in mind. This imposes certain restrictions on the algorithm that are irrelevant for a software implementation.

OoOJava [Jenista et al. 2011] uses heap dependence queues. These queues operate using generations as done in this work. It is, however, more complex, as generations may be organized as hash tables, where each bucket in the hash table holds a chain of dependent tasks. Chains in different buckets may execute concurrently.

Best et al. [2011] present a generation-based scheme with one program-wide queue. Tasks are added to a generation when they are proven to have nonoverlapping memory footprints; otherwise, a new generation is started. The scheduler processes one generation at a time. This, however, limits parallelism when generations are small and is prone to unbalance within generations.

Gupta and Sohi [2011] present a scheme where each object has a wait-list of tasks that will operate on that object. This is a variation on the list scheme. However, their scheme requires that tasks with an input dependence issue in program order (although they may complete in any order). This may reduce parallelism measurably. This restriction is necessary because they do not move tasks in the oldest (ready) generation to a ready list.

Instead of following edges in a graph, some schemes scan the list of all spawned tasks to find common arguments [Kurzak and Dongarra 2009]. This consumes time proportional to the size of the task graph and appears necessary only for handling aliased task arguments [Perez et al. 2010].

\section{CONCLUSION}

This article studies data structures and algorithms for the efficient implementation of task dataflow scheduling. Efficiency is key in the context of strong scaling, where scheduler overheads must be minimized. Our algorithms are based on the notions of versions and generations of objects. We defined schemes based on graphs, hypergraphs, and lists. We also presented an optimization to embed a linked list of tasks in the task representation, which dramatically reduces runtime system overhead. Besides the three edge-centric schemes, we analyzed an edgeless scheme based on ticket locks. 
An unanticipated result of our evaluation is that a straightforward implementation of the task graph as a graph is susceptible to bad scaling behavior. This is true if the language supports annotations such as commutative or reduction operands.

Evaluation with microbenchmarks consistently showed that the edgeless tickets scheme outperforms the edge-centric schemes. The tickets scheme allows singleargument task spawns in as little as $0.20 \mu \mathrm{s}$ and is at least 5 to $10 \%$ faster than the best edge-centric scheme. Some experiments reveal substantially larger improvements.

The tickets scheme has one shortcoming, which is in the design of the task pool. The task pool holds all pending tasks and is searched for ready tasks by idle processors. It is organized as a hash table where the depth of a task in the task graph is used as a key to speed up searches. In contrast, edge-centric schemes are able to locate ready tasks more efficiently. The task pool, however, only adds latency to the scheduler when it holds a large task graph. This is not always the case in our scheduler.

It is an open question whether the edgeless ticket scheme can be extended to store successor tasks efficiently, without incurring the overhead of the edge-centric schemes. Such a scheme could avoid the need for a task pool.

In future work, we will study dependence tracking in the context of array sections, which would lead to partially overlapping address ranges. Schemes exist that allow this [Tzenakis et al. 2012; Perez et al. 2010], or where uncertainty in the address ranges plays a role [Best et al. 2011; Jenista et al. 2011]. The overheads for these schemes have been reported as substantial, up to milliseconds per task spawn.

\section{REFERENCES}

Agrawal, K., Leiserson, C. E., And SukHa, J. 2010. Executing task graphs using work-stealing. In Proceedings of the 24th IEEE International Parallel and Distributed Processing Symposium (IPDPS'10). 1-12.

Alvanos, M., Tzenakis, G., Bilas, A., and Nikolopoulos, D. S. 2011. Design and evaluation of a task-based parallel H.264 video encoder for heterogeneous processors. In Proceedings of SAMOS XI: International Conference on Embedded Computer Systems: Architectures, Modeling and Simulation. 217-224.

Augonnet, C., Thibault, S., NAmyst, R., And Wacrenier, P.-A. 2010. StarPU: A unified platform for task scheduling on heterogeneous multicore architectures. Concurrency and Computation: Practice and Experience $23,2,187-198$.

Barcelona Supercomputing Center. 2008. SMP Superscalar (SMPSS) User's Manual, 2.2 ed. Barcelona Supercomputing Center.

Berge, C. 1973. Graphs and Hypergraphs. North-Holland.

Best, M. J., Mottishaw, S., Mustard, C., Roth, M., Fedorova, A., and Brownsword, A. 2011. Synchronization via scheduling: Techniques for efficiently managing shared state. In Proceedings of the 32nd ACM SIGPLAN Conference on Programming Language Design and Implementation. 640-652.

BIEnIA, C. 2011. Benchmarking Modern Multiprocessors. PhD Dissertation, Princeton University.

Bosilca, G., Bouteiller, A., Danalis, A., Herault, T., Lemarinier, P., and Dongarra, J. 2010. DAGuE: A Generic Distributed DAG Engine for High Performance Computing. Technical Report. Innovative Computing Laboratory.

Budimlić, Z., Burke, M., Cavé, V., Knobe, K., Lowney, G., Newton, R., Palsberg, J., Peixotto, D., Sarkar, V., Schlimbach, F., and Taşirlar, S. 2010. Concurrent collections. Sci. Program. 18, 3-4, 203-217.

Chan, E., Quintana-Orti, E. S., Quintana-Orti, G., and van de GeiJn, R. 2007. Supermatrix out-of-order scheduling of matrix operations for SMP and multi-core architectures. In Proceedings of the 19th Annual ACM Symposium on Parallelism in Architectures and Applications. 116-125.

ChI, C. C. AND JuURLINK, B. 2011. A QHD-capable parallel H.264 decoder. In Proceedings of the International Conference on Supercomputing. 317-326.

Conover, W. J. AND Iman, R. L. 1981. Rank transformations as a bridge between parametric and nonparametric statistics. American Statistician 35, 3, 124-129.

Duran, A., Ayguadé, E., Badia, R. M., Labarta, J., Martinell, L., Martorell, X., and Planas, J. 2011. OmpSs: A proposal for programming heterogeneous multi-core architectures. Parallel Processing Letters 21, 2 , 173-193

DongarRa, J., Beckman, P., ET AL. 2011. The international exascale software project roadmap. International Journal of High Performance Computer Applications 25, 1, 3-60. 
Frigo, M., HalPern, P., Leiserson, C. E., AND Lewin-Berlin, S. 2009. Reducers and other Cilk++ hyperobjects. In Proceedings of the 21st Annual Symposium on Parallelism in Algorithms and Architectures. 79-90.

Frigo, M., Leiserson, C. E., And Randall, K. H. 1998. The implementation of the Cilk-5 multi-threaded language. In Proceedings of the 1998 ACM SIGPLAN Conference on Programming Language Design and Implementation. 212-223.

Gupta, G. And Sohi, G. S. 2011. Dataflow execution of sequential imperative programs on multicore architectures. In Proceedings of the 44th Annual IEEE/ACM International Symposium on Microarchitecture. 59-70.

Hennessy, J. L. And Patterson, D. A. 2003. Computer architecture: A Quantitative Approach, 3rd ed. Morgan Kaufmann.

Jenista, J. C., Eom, Y. H., AND Demsky, B. C. 2011. OoOJava: Software out-of-order execution. In Proceedings of the 16th ACM Symposium on Principles and Practice of Parallel Programming. 57-68.

Kurzak, J. AND Dongarra, J. 2009. Fully Dynamic Scheduler for Numerical Computing on Multicore Processors. Technical Report UT-CS-09-643. LAPACK Working Note 220.

Perez, J. M., BADIA, R. M., AND LABARTA, J. 2008. A dependency-aware task-based programming environment for multicore architectures. In Proceedings of the IEEE International Conference on Cluster Computing (CLUSTER'08). 142-151.

Perez, J. M., Badia, R. M., and Labarta, J. 2010. Handling task dependencies under strided and aliased references. In Proceedings of the International Conference on Supercomputing. 263-274. Retrieved from http://dx.doi.org/10.1145/1810085.1810122.

Tzenakis, G., Papatriantafyllou, A., Kesapides, J., Pratikakis, P., Vandierendonck, H., and Nikolopoulos, D. S. 2012. BDDT: Block-level dynamic dependence analysis for deterministic task-based parallelism. In Proceedings of the 17th ACM SIGPLAN Symposium on Principles and Practice of Parallel Programming. 301-302. Retrieved from http://dx.doi.org/10.1145/2145816.2145864.

Vandierendonck, H., Chronaki, K., And Nikolopoulos, D. S. 2013. Deterministic Scale-Free Pipeline Parallelism with Hyperqueues. In Proceedings of Supercomputing'13: High-Performance Computing, Networking, Storage and Analysis. 32:1-32:12. Retrieved from http://dx.doi.org/10.1145/2503210.2503233.

Vandierendonck, H., Pratikakis, P., and Nikolopoulos, D. S. 2011a. Parallel programming of general-purpose programs using task-based programming models. In Proceedings of the 3rd USENIX Workshop on Hot Topics in Parallelism (HotPar'11).

Vandierendonck, H., Tzenakis, G., And Nikolopoulos, D. S. 2011b. A unified scheduler for recursive and task dataflow parallelism. In Proceedings of the 20th International Conference on Parallel Architectures and Compilation Techniques. 1-11.

Received June 2013; revised November 2013; accepted November 2013 